\section{Sepsis temprana en un recién nacido pretérmino por Pantoea agglomerans: informe de caso y revisión de la literatura}

Gálvez-Cuitiva EA ${ }^{1}$, Peralta-Palmezano $\mathrm{JJ}^{2}$, Lonngi-Rojas $\mathrm{G}^{3}$

\begin{abstract}
Resumen
INTRODUCCIÓN: Pantoea Agglomerans es un bacilo gramnegativo miembro de la familia Enterobacteriaceae, de distribución ubicua que, bajo ciertas circunstancias, puede tener un comportamiento patógeno en el ser humano. Son pocos los casos de infección neonatal no asociados al cuidado de la salud descritos en la literatura; ninguno de ellos reportado en Colombia.

PRESENTACIÓN DEL CASO: niña recién nacida, pretérmino, que desde su primer día presentó una inadecuada adaptación neonatal, con dificultad respiratoria, sepsis y un rápido deterioro clínico, lo cual requirió manejo y soporte en la unidad de cuidados intensivos neonatales. Se aisló por hemocultivos $P$. agglomerans, susceptible al manejo antibiótico instaurado, con una respuesta adecuada, así como una evolución clínica favorable.
\end{abstract}

CONCLUSIÓN: la sepsis neonatal por $P$. agglomerans es poco frecuente, por lo que su desenlace dependerá de muchos factores: la detección temprana, la asistencia oportuna y el manejo adecuado que se brinde.

PALABRAS CLAVE: Pantoea agglomerans, sepsis temprana, recién nacido, pretérmino.

Acta Pediatr Mex. 2018 Jan;39(1):52-59.

\section{Early Sepsis in a Preterm Newborn by Pantoea agglomerans: Case Report and Literature Review.}

Gálvez-Cuitiva EA ${ }^{1}$, Peralta-Palmezano $\mathrm{JJ}^{2}$, Lonngi-Rojas $\mathrm{G}^{3}$

\begin{abstract}
INTRODUCTION: Pantoea agglomerans is a gram-negative bacillus member of the Enterobacteriaceae family, of ubiquitous distribution and in certain circumstances can have a pathogenic behavior in humans, with few cases of neonatal infection not associated with health care described in the literature, none of them reported in Colombia.

CASE PRESENTATION: In the following report, we present the case of a preterm, female newborn who, from the first day presents an
\end{abstract}

${ }^{1}$ Perinatología-Neonatología. Dpto. Pediatría, Facultad de Medicina. Instituto Materno Infantil.

${ }^{2}$ Dpto. Pediatría, Facultad de Medicina.

${ }^{3}$ Coordinador Académico, División de Perinatología y Neonatología, Dpto. Pediatría, Facultad de Medicina, Director Dpto. Pediatría y Neonatología Clínica Marly.

Universidad Nacional de Colombia.

Recibido: 15 de marzo del 2017

Aceptado: 7 de julio del 2017

Correspondencia

Eduardo Gálvez-Cuitiva

egalvezc@unal.edu.co

\section{Este artículo debe citarse como}

Gálvez-Cuitiva EA, Peralta-Palmezano JJ, LonngiRojas G. Sepsis temprana en un recién nacido pretérmino por Pantoea agglomerans: informe de caso y revisión de la literatura. Acta Pediatr Mex. 2018;39(1):52-59. 
inadequate neonatal adaptation, with respiratory distress, sepsis and a rapid clinical deterioration, which required management and support in the neonatal intensive care unit. It was isolated by blood cultures $P$. agglomerans, susceptible to the antibiotic management established, with a suitable posterior response, as well as a favorable clinical evolution.

CONCLUSION: Neonatal sepsis due to $P$. agglomerans is uncommon, and its outcome will depend on many factors, as well as early detection, timely assistance and appropriate management.

KEYWORDS: Pantoea agglomerans; early-onset sepsis; newborn; preterm

\section{Correspondence \\ Gabriel Lonngi-Rojas \\ glonngir@unal.edu.co}

\section{INTRODUCCIÓN}

El Pantoea agglomerans es un bacilo gramnegativo, no encapsulado, no esporulado, perteneciente a la familia Enterobacteriaceae, descrito inicialmente en plantas, aislado posteriormente en animales y por primera vez en humanos en $1965 .{ }^{1}$ Conocido previamente como Erwinieae herbicola o Enterobacter agglomerans, se agrupó posteriormente en un solo género: Pantoea; ${ }^{2}$ de los cuales se han descrito siete especies: P. agglomerans, P. ananatis, P. citrea, P. dispersa, P. punctata, $P$. stewartii y $P$ terrea. No obstante, con la tipificación multilocus de secuencias (MLST, por sus siglas en inglés) se ha sugerido la existencia de diez potenciales especies nuevas relacionadas filogenéticamente.,

El P. agglomerans es capaz de crecer de manera óptima a $25^{\circ} \mathrm{C}$; por lo tanto, es de naturaleza ubicua, aislado de diversas fuentes geográficas y ecológicas, comúnmente en superficies de plantas, frutas, vegetales, en el suelo y heces animales o humanas. ${ }^{5,6}$ Se considera un organismo saprófito para el hombre, pero que bajo ciertas condiciones, puede desempeñar un papel patógeno.

En la literatura se ha descrito como causante de infecciones localizadas por traumatismo con plantas, pinchazos con espinas o astillas; puede presentar: artritis séptica, ${ }^{5,7-9}$ sinovitis, ${ }^{10-12}$ osteítis tibial, ${ }^{13}$ absceso cerebral, ${ }^{14}$ endoftalmitis, ${ }^{15,16}$ infección urinaria, ${ }^{5}$ abscesos cutáneos ${ }^{5}$ y peritonitis, entre otros, en pacientes a quienes se les han instalado dispositivos invasivos. ${ }^{17-19}$ Adicionalmente, el $P$. agglomerans se ha relacionado con infecciones asociadas al cuidado de la salud, se encuentra como contaminante de nutriciones parenterales, ${ }^{20,21}$ anestésicos, ${ }^{22}$ hemoderivados usados en transfusión, ${ }^{23}$ en torundas de algodón, ${ }^{24}$ leche de fórmula ${ }^{25}$ y catéteres venosos. ${ }^{5,26}$

Los primeros casos de sepsis neonatal por Pantoea spp fueron secundarios a contaminación de soluciones intravenosas. ${ }^{27}$ Son pocos los casos de infección neonatal por P. agglomerans no asociados al cuidado de la salud reportados en la literatura, de acuerdo a la revisión, en Colombia no se han documentado casos.

En el presente reporte describimos un caso de sepsis neonatal temprana por $P$. agglomerans en un recién nacido pretérmino tardío, obtenido por vía vaginal, no asociada al cuidado de la salud.

\section{PRESENTACIÓN DE CASO}

Recién nacido, sexo femenino, producto de primera gestación, madre de 16 años, con estudios prenatales: antígeno de superficie de virus de 
hepatitis B negativo, serologías IgM para rubéola, citomegalovirus y toxoplasma no reactivos. A las 10 semanas de gestación prueba rápida para VIH negativa y serología VDRL no reactivo en el preparto; sin estudio para estreptococo del grupo B.

Inició trabajo de parto prematuro espontáneo, amniotomía intraparto con evidencia de líquido amniótico meconial espeso. Se obtuvo producto femenino prematuro de 35 semanas de gestación calculado por el método de Capurro, vía vaginal en hospital de segundo nivel de atención, con peso al nacer de $2340 \mathrm{~g}$ y talla de $45 \mathrm{~cm}$, adecuados para edad gestacional. Al nacimiento requirió adaptación neonatal conducida inmediata, pinzamiento de cordón habitual, aspiración de meconio en orofaringe, oxigenoterapia asistida y posterior intubación orotraqueal e inició de ventilación mecánica invasiva, Apgar de 4 al minuto (frecuencia cardiaca mayor de 100 -2-, esfuerzo respiratorio irregular -1-, escasas muecas $-1-$, sin tono muscular y cianosis generalizada) y 6 a los 5 minutos (frecuencia cardiaca mayor de 100 -2-, esfuerzo respiratoria irregular -1-, extremidades flexionadas -1-, acrocianosis -1-). Se realizó diagnóstico de síndrome de aspiración de meconio (SAM), se instauró manejo con surfactante exógeno endotraqueal.

En su primer día de vida los estudios paraclínicos muestran: hemograma: leucocitos: $16,300 / \mathrm{mm}^{3}$, neutrófilos: 5216/mm³ $\mathrm{Hb}: 21 \mathrm{~g} / \mathrm{dL}, \mathrm{Htco}: 64 \%$. Plaquetas: $143,000 / \mathrm{mm}^{3}$, proteína C reactiva: $15.8 \mathrm{mg} / \mathrm{dL}$ (referencia menor de $0.6 \mathrm{mg} / \mathrm{dL}$ ).

Se realizó diagnóstico presuntivo de sepsis neonatal temprana y se inició manejo antibiótico de acuerdo a guías institucionales con ampicilina (100 miligramos por kilogramo de peso al día) y gentamicina (5 miligramos por kilogramo de peso al día) previa toma de hemocultivos. Durante su evolución presentó deterioro hemodinámico por hipotensión, bradicardia, oligoanuria, por lo que se inició soporte vasopresor con dopamina y noradrenalina, deterioro respiratorio que llevó a uso de ventilación invasiva con uso de ventilación mecánica convencional y traslado a un hospital de tercer nivel de atención.

Al segundo día de vida se tomaron estudios paraclínicos de control: hemograma con leucocitos: 4490/ $\mathrm{mm}^{3}$, neutrófilos: $2873 / \mathrm{mm}^{3}$, linfocitos: 58 / mm33 $\mathrm{Hb}: 17.7 \mathrm{~g} / \mathrm{dL}$, Htco: 50.6\%. Plaquetas: $87,000 / \mathrm{mm}^{3}$ (control del tercer día: 7000 células/ $\mathrm{mm}^{3}$ ), proteína $\mathrm{C}$ reactiva: $16.41 \mathrm{mg} / \mathrm{dL}$, TGO: $146 \mathrm{U} / \mathrm{L}, \mathrm{TGP}: 27, \mathrm{U} / \mathrm{L}$ creatinina: $0.92 \mathrm{mg} / \mathrm{dL}$, nitrógeno ureico en sangre: $21 \mathrm{mg} / \mathrm{dL}$, bilirrubinas total: $7.94 \mathrm{mg} / \mathrm{dL}$, directa: $1.33 \mathrm{mg} / \mathrm{dL}$, indirecta: $6.61 \mathrm{mg} / \mathrm{dL}$, tiempo de protrombina: 40.9 segundos (control: 13.8 segundos), INR: 3.44, tiempo de tromboplastina parcial: 79.9 segundos (control: 28.9 segundos), gasometría: $\mathrm{pH}$ 6.99, $\mathrm{PCO}_{2}: 70 \mathrm{mmHg}$, BE: -15.5, HCO3: $16.9 \mathrm{mg} / \mathrm{dL}$, lactato sérico: 2,5.

En hemocultivos periféricos tomados en el primer día de vida hay crecimiento de $P$. agglomerans, sensible a ampicilina, gentamicina, amoxicilina/ clavulanato, amikacina, piperacilina/tazobactam, trimetoprim/sulfametoxazol, imipenem; sensibilidad intermedia a ertapenem y levofloxacina y resistencia a ciprofloxacina, ceftriaxona, cefuroxima, cefepime, cefoxitin.

Paciente con falla multisistémica de origen séptico, con compromiso hemodinámico que requirió soporte con dopamina, dobutamina y norepinefrina, ecocardiograma de ingreso sin alteraciones estructurales, falla renal de característica pre renal que mejora paulatinamente con reanimación hídrica; compromiso respiratorio desde su ingreso con acidemia respiratoria corregida en las primeras 24 horas, seguido de hemorragia pulmonar, uso de ventilación de alta frecuencia oscilatoria y óxido nítrico por 96 horas, con posterior evolución favorable, egresando de unidad neonatal sin requerimiento de oxígeno complementario. Falla hepática que conllevó a trasfusión de plasma fresco congelado en 4 ocasiones, con mejoría paulatina de 
tiempos de coagulación, falla hematológica con plaquetopenia sumada a datos de sangrado pulmonar, recibiendo transfusión de concentrado de plaquetas y glóbulos rojos. No presentó convulsiones, ultrasonido transfontanelar a su ingreso y al egreso de unidad de intensivos, sin alteraciones, requirió sedación con morfina durante los 4 primeros días con posterior descenso gradual de ésta, apoyado con nutrición parenteral desde el primer día de vida y durante 16 días, con cambio progresivo a nutrición enteral, egresando con apego a seno materno sin complicaciones.

En general evolución clínica favorable, completó tratamiento antibiótico de 10 días ampicilina y 5 días gentamicina, con dos hemocultivos de control negativos a los 7 y 18 días de vida. Completó estancia por 17 días en unidad de cuidado intensivo, egresando con peso de 2405 gramos, con seguimiento ambulatorio por el programa de plan canguro institucional a los tres meses de seguimiento, con adecuada ganancia de peso, sin afectación en neurodesarrollo. No ha requerido nuevas hospitalizaciones.

\section{DISCUSIÓN}

De acuerdo a las cifras reportadas en la literatura, actualmente la mortalidad neonatal representa el $40 \%$ de toda la mortalidad infantil. ${ }^{28}$ Cada año se registran cerca de 2,6 millones de muertes neonatales; tres cuartas partes de estas ocurren en la primera semana de vida y la mayoría (99\%) en los países de ingresos bajos y medios. ${ }^{28,29} \mathrm{La}$ sepsis neonatal temprana es la tercera causa de mortalidad neonatal (después de la prematuridad y la asfixia perinatal), responsable del $13 \%$ de toda la mortalidad neonatal y del $42 \%$ de las muertes en la primera semana de vida. ${ }^{30}$

La sepsis neonatal se define como un síndrome clínico caracterizado por signos y síntomas de infección, con o sin bacteriemia, en el primer mes de vida y se clasifica dependiendo del momento de inicio de la infección y en el supuesto modo de transmisión, en sepsis temprana y tardía.

La sepsis temprana se define por el inicio de la infección en las primeras 72 horas de vida, debido a la transmisión intraparto. Es causada por organismos prevalentes en el tracto genital materno. La sepsis tardía inicia después de 72 horas, la mayoría de las veces causada por agentes patógenos adquiridos en el ambiente externo del hogar o el hospital; es una complicación común de la estancia prolongada en la unidad de cuidado intensivo neonatal. ${ }^{31}$ Los microorganismos más comúnmente asociados con el inicio temprano de la infección son el Streptococcus agalactiae, Escherichia coli, Streptococcus viridans, Lysteria monocytogenes y Klebsiella sp. Luego de las 72 horas los gérmenes más comunes son el estafilococo coagulasa negativo, E. coli, S. aureus, Candida albicans, Klebsiella sp., Enterococccus sp., Pseudomonas aeruginosa. ${ }^{32-35}$

La infección por $P$. agglomerans es rara en humanos ya que en general es considerada un microorganismo no invasivo. Sin embargo, si se introduce directamente en la sangre es tan virulento como cualquier otra bacteria, ${ }^{36}$ principalmente en pacientes susceptibles, con cierta predilección por los pulmones cuando está implicado en infecciones sistémicas. ${ }^{37}$

El aislamiento de $P$. agglomerans en sepsis neonatal no asociada al cuidado de la salud es poco frecuente. Los casos descritos de infección neonatal por $P$. agglomerans se han presentado en neonatos a término y pretérminos, ya sea como sepsis tardía o temprana..$^{36-44}$ Los primeros casos reportados se asociaron a brotes por contaminación de infusiones para nutrición parenteral. ${ }^{38}$ Aunque se han descrito infecciones por otros tipos de Pantoea, ${ }^{45}$ la infección por $P$. agglomerans es la más frecuente. En el Cuadro 1 se resumen los casos reportados de sepsis neonatal por esta bacteria con las principales características y se comparan con el caso presentado. 


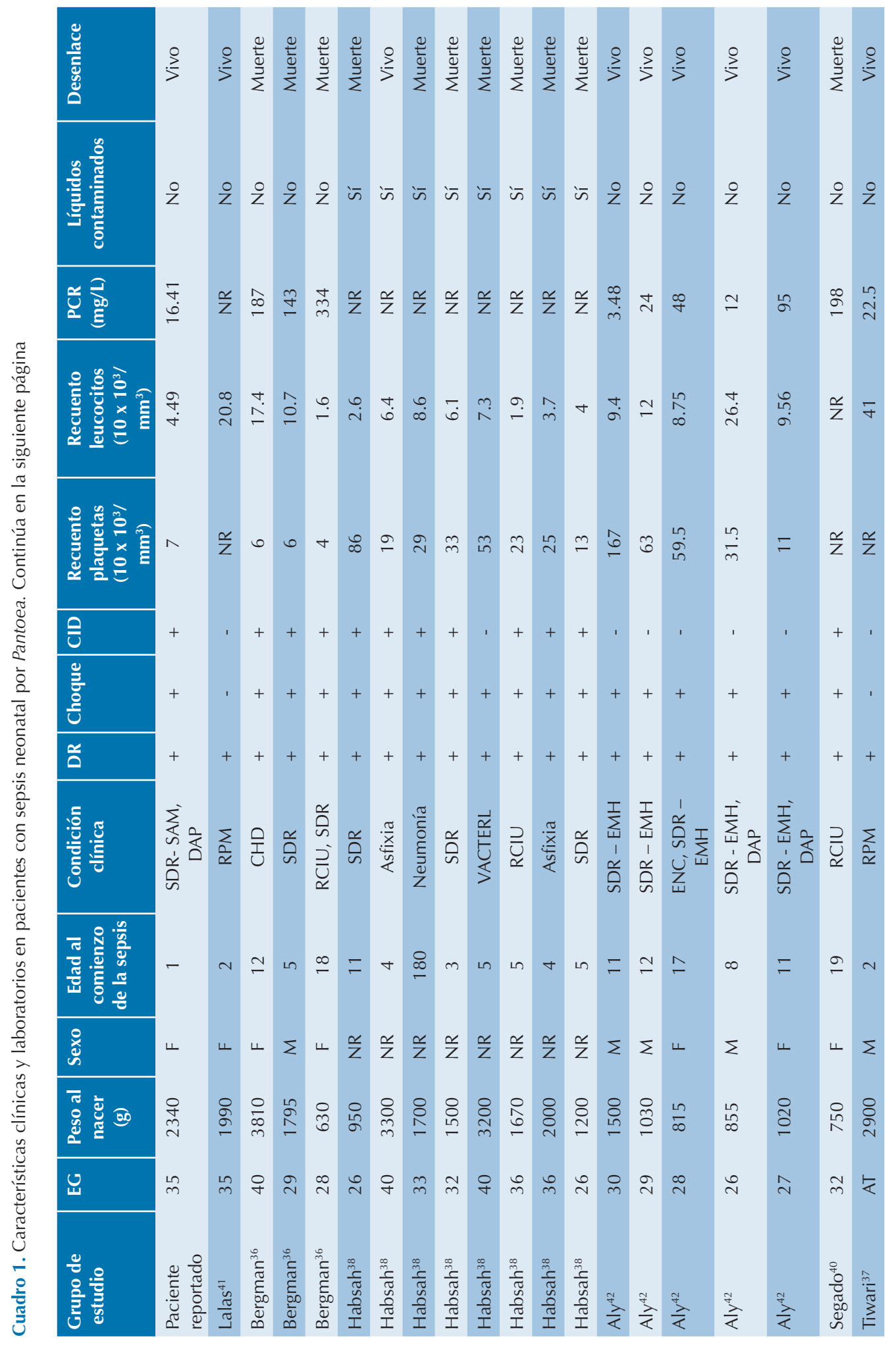




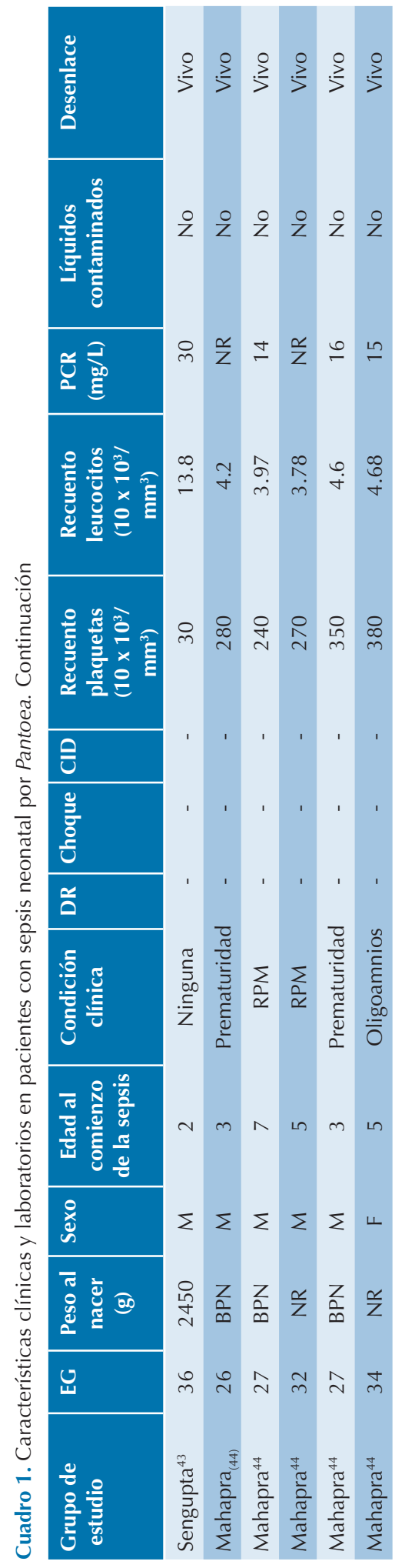

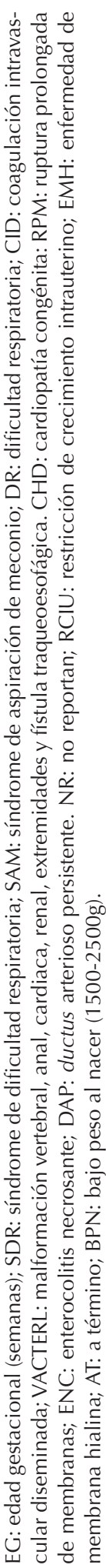

Aunque no se han establecido los mecanismos de infección de la $P$. agglomerans, se ha descrito una transmisión vertical en reportes de casos de sepsis neonatal temprana ${ }^{37,41-44}$ con pronóstico más favorable, en comparación con aquellos casos asociados al cuidado de la salud, a pesar de un adecuado tratamiento antibiótico. ${ }^{38}$ Lo anterior, puede ser debido el mayor inóculo directamente al torrente sanguíneo de los pacientes, cepas más virulentas, la inmunodeficiencia de los neonatos pretérmino y fuentes de infección no controladas en aquellos casos asociados al cuidado de la salud. ${ }^{39}$

La forma probable de infección de este paciente fue durante el periparto, como único factor de riesgo prematuridad, no se logró aislamiento de $P$. agglomerans en cultivo de muestra rectovaginal en la madre. La presentación clínica inicial se caracterizó por una inadecuada adaptación neonatal, con un síndrome de dificultad respiratoria que requirió soporte ventilatorio y una progresión a falla multisistémica secundaria al choque séptico, además de trombocitopenia y posterior CID, consistente con otros reportes y series de casos en los cuales se reportan trombocitopenia hasta en un $75 \%$ de los casos, ${ }^{36,39,40,42}$ con aislamiento en dos hemocultivos de sangre periférica de distinto sitio de punción a las 15 horas de vida.

La mayoría de las veces la Pantoea spp. es sensible a los antibióticos parenterales de uso común. El organismo aislado en los hemocultivos de este paciente fue susceptible a la mayoría de los antibióticos, por lo cual se continuó el antibiótico instaurado empíricamente, de acuerdo a las guías institucionales (ampicilina a dosis de 100 miligramos por kilogramo de peso cada doce horas y gentamicina 5 miligramos por kilogramo de peso al día), con una adecuada respuesta a los mismos.

No hay un consenso ni suficiente evidencia sobre qué antibiótico iniciar en la infección por 
este germen por lo que, teniendo en cuenta los resultados obtenidos, se recomienda dar manejo antibiótico empírico de primera línea para sepsis neonatal temprana con ampicilina y aminoglucósido ${ }^{46}$ y, dependiendo del antibiograma y la respuesta clínica, dirigir el tratamiento.

Al relacionar la edad gestacional con la evolución clínica y la respuesta al manejo se han encontrado resultados contradictorios en los estudios reportados en la literatura, en donde en algunos se ha asociado con peores desenlaces en los recién nacidos pretérminos, ${ }^{36,40}$ mientras que en otros se presentaba supervivencia de los pacientes reportados, la mortalidad en la serie de casos más grande es del $87.5 \% .^{37,38,41-44}$ Sin embargo, dado el tipo de los estudios observacionales analizados (reportes de casos y series de casos), se requiere de estudios con mayor cantidad de población y de mejor diseño para determinar la verdadera prevalencia e impacto de esta infección en los recién nacidos, así como plantear medidas preventivas y de mejora en la calidad de atención. En este caso, de un recién nacido pretérmino con sepsis por $P$. agglomerans, a pesar de todas las complicaciones asociadas durante el curso de su proceso infeccioso y estancia en la unidad de cuidados intensivos neonatales, presentó una evolución clínica favorable y una respuesta satisfactoria al tratamiento.

\section{CONCLUSIONES}

La sepsis neonatal por $P$. agglomerans es infrecuente, con manifestaciones clínicas variables y cuyo desenlace dependerá de muchos factores: edad gestacional del paciente, modo de transmisión de la enfermedad, así como lo oportuno del tratamiento antibiótico y de soporte. Por lo tanto, la identificación temprana, junto con un tratamiento guiado por la clínica y por pruebas de susceptibilidad antibiótica, son esenciales para un manejo adecuado y para resultados favorables. Del mismo modo, realizar un ade- cuado cuidado y control antenatal de la gestante, mantener medidas de bioseguridad estandarizadas y el adecuado manejo de productos de uso sanitario, así como otras políticas para disminuir las infecciones, como lo son el uso racional de antibióticos, son necesarios para la prevención de estos casos.

\section{REFERENCIAS}

1. Muraschi T, Friend M, Bolles D. Erwinia-Like Microorganisms Isolated from Animal and Human Hosts. Applied Microbiology. 1965;13(2):128-131.

2. Walterson, A., Stavrinides, J., Pantoea: insigihts into a highly versatile ande diverse genus within the Enterobacteria. FEMS Microbiology Reviews. 2015;fuv 027,39:968-984.

3. Deletoile A, Decre D, Courant S, Passet V, Audo J, Grimont P, et al. Phylogeny and Identification of Pantoea Species and Typing of pantoea agglomeransstrains by multilocus gene sequencing. J Clin Microbiol. 2009;47(2):300-10.

4. Brady C, Cleenwerckl, Venter S, et al. Phylogeny and identification of Pantoea species associated with plants, humans and the natural environment based on multilocus sequence analysis (MLSA). Syst Appl Microbiol. 2008;31:447-60.

5. Cruz A, Cazacu A, Allen C. Pantoeaagglomerans, a plant pathogen causing human disease. J Clin Microbiol. 2007;45(6):1989-92.

6. ndersson A, Weiss N, Rainey F, Salkinoja-Salonen M. Dustborne bacteria in animal sheds, schools and children's day care centres. J Appl Microbiol. 1999;86:622-34.

7. Flatauer F, Khan M. Septic arthritis caused by Enterobacteragglomerans. Arch Intern Med.1978;138:788.

8. Kratz A, Greenberg D, Barki Y, Cohen E, Lifshitz M. Pantoea agglomerans as a cause of septic arthritis after palm tree thorn injury; case report and literature review. Arch Dis Child. 2003;88:542-544.

9. De Champs C, Le Seaux S, Dubost JJ, Boisgard S, Sauvezie $B$, Sirot J. Isolation of Pantoea agglomerans in two cases of septic monoarthritis after plant thorn and wood sliver injuries. J Clin Microbiol. 2000;38:460-461.

10. Stromqvist B, Edlund E, Lidgren L. A case of blackthorn synovitis. Acta Orthop Scand. 1985;56:342-343.

11. Oleginski TP, Bush DC, Harrington TM. Plant thorn synovitis: an uncommon cause of monoarthritis. Semin Arthritis Rheum. 1991;21:40-46.

12. Duerinckx J. Subacute Synovitis of the Knee After a Rose Thorn Injury Unusual Clinical Picture. Clin Orthop Relat Res. 2008;466:3138-3142.

13. Laporte C, Demachy MC, Thevenin-Lemoine C: Tibialosteitis caused by Pantoea agglomerans after open grade IIIB tibial shaft fracture (in French). Rev Chir Orthop Reparatrice Appar Mot. 2002;88:625-627. 
Gálvez-Cuitiva EA et al. Sepsis temprana por Pantoea agglomerans

14. Wechsler A, et al. Brain Abscess Caused by an Erwinia Species. Report of a Case and Review of the Literature Am J Med. 1971;51(5):680-684.

15. Mason GI, Bottone E, Podos SM.Traumatic Endophthalmitis Caused by an Erwinia Species. Am J Ophthalmol.1976;82(5):709-13.

16. Seok S. A Case of Bilateral endogenous Pantoea Agglomerans Endophthalmitis with Interstitial Lung Disease. Korean J Ophthalmol. 2010;24(4): 249-251.

17. Kahveci A, Asicioglu E, Tigen E, Ari E, Z et al. Unusual causes of peritonitis in a peritoneal dialysis patient: Alcaligenesfaecalisand Pantoea agglomerans. Ann Clin Microbiol Antimicrob. 2010;10:12-4.

18. Lim PS, Chen SL, Tsai CY, Pai MA. Pantoea-peritonitis in a patient receiving chronic ambulatory peritoneal dialysis. Nephrology. 2006;11:97-9.

19. Magnette C, Tintillier M, Horlait G, Cuvelier C, Pochet JM. Severe peritonitis due to Pantoea agglomerans in a CCPD patient. Perit Dial Int. 2008;28:207-8.

20. Panknin H-T. An outbreak of fatal pantoeainfections in newborn infants, caused by contaminated infusion solutions. Kinderkranken schwester. 2006;25(5):189-90.

21. Maki DG, Rhame FS, Mackel DC, et al. Nationwide epidemic of septicemia caused by contaminated intravenous products. I. Epidemiologic and clinical features. Am J Med. 1976;60:471-85.

22. Bennet SN, McNeil MM, Lee MP, Arduino M, Villarino E, Perrota Dennis, et al. Postoperative infections traced to contamination of an intravenous anesthetic, propofol. $\mathrm{N}$ Eng J Med. 1995;3333:147-154.

23. Arduino MJ, Bland LA, Tipple MA, et al. Growth and endotoxin production of Yersinia enterocolitica and Enterobacter agglomerans in packed erythrocytes. J ClinMicrobiol.1989;27:1483-85.

24. Koo HS, Kim JS, Eom JS. Pseudo outbreak of Pantoea species bacteremia associated with contaminated cotton pledgets. Am J Inf Control. 2006;34:443-446.

25. Mardaneh J, Dallal MMS. Isolation, identification and antimicrobial susceptibility of Pantoea (Enterobacter) agglomerans isolated from consumed powdered infant formula milk (PIF) in NICU ward: First report from Iran. Iran J Microbiol. 2013;5(3):263-267.

26. Bicudo EL, Macedo VO, Carrara MA, Castro FF, Rage RI. Nosocomial outbreak of Pantoea agglomerans in a pediatric urgent care center. Braz J Infect Dis. 2007;11:281-4.

27. Matsaniotis NS, Syriopoulou VP, Theodoridou MC, Tzanetou KG. Enterobacter sepsis in infants and children due to contaminated intravenous fluids. Infect Control. 1984;5:471-7.

28. Wang H, Liddell CA, Coates MM, Mooney M., Levitz C, Schumacher A. et al.Global, regional, and national levels of neonatal, infant, and under-5 mortality during 1990-2013: a systematic analysis for the Global Burden of Disease Study 2013. Lancet. 2014;384:957-79.

29. Lawn JE, Cousens S, Zupan J, et al.4 million neonatal deaths: when? Where? Why? Lancet. 2005;365:891-900.

30. Liu L, Johnson HL, Cousens S, Perin J, Scott S. Lawn J, et al.Global, regional, and national causes of child mortality: an updated systematic analysis for 2010 with time trends since 2000. Lancet. 2012;379:2151-61.

31. Stoll BJ, Hansen N, Fanaroff AA, Wright LL, Carlo WA, Ehrenkranz RA, et al. Late- onset sepsis in very low birth weight neonates: the experience of the NICHD Neonatal Research Network. Pediatrics. 2002;110:285-91.

32. Klinger G, Levy I, Sirota L, Boyko V, Reichman B, LernerGeva L. Epidemiology and risk factors for early onset sepsis among very-low-birthweight infants. Am J Obstet Gynecol. 2009;201:38.e1-6.

33. Shah BA,Padbury JF. Neonatalsepsis: an old problem with new insights. Virulence. 2014;5(1):170-178.

34. Zaidi AKM, Thaver D, Ali SA, et al. Pathogens associated with sepsis in newborns and young infants in developing countries. Pediatr Infect Dis J. 2009;28:S10-S18.

35. Stoll BJ, Hansen NI, Sánchez PJ, et al. Early onset neonatal sepsis: the burden of group B Streptococcal and E. coli disease continues. Pediatrics. 2011;127:817-26.

36. Bergman K, Arends J, Schölvinc E. Pantoea agglomerans septicemia in three newborn infants. The pediatric Infectious Disease Journal. 2007;26(5):453-454.

37. Tiwari S, Beriha S. Pantoea species causing early onset neonatal sepsis: a case report. Journal of Medical Case Reports. 2015;9:188-190.

38. Habsah H., Zeehaida M, Van Rostenberghe $H$, Noraida R, Wan Pauzi W, Fatimah I, Rosliza A, NikSharimah N. H. Maimunahc An outbreak of Pantoea spp. in a neonatal intensive care unit secondary to contaminated parenteral Nutrition. Journal of Hospital Infection. 2005;61:213-218.

39. Van Rostenberghe $\mathrm{H}$, Wan Pauzi WI, Habsha $\mathrm{H}$, et al. The clinical picture of neonatal infection with Pantoea species. Jpn J Infect Dis. 2006;59:120-121.

40. Segado A. Alonso A. Lubián S, García A. Pantoeaagglomerans: ¿un nuevo patógeno en la unidad de cuidados intensivos neonatales? Arch Argent Pediatr. 2012;110(4):e77-e79

41. Lalas KM, Erichsen D. Sporadic Pantoea agglomerans bacteremia in a near-term female: case report and review of literature. Jpn J Infect Dis. 2010;63:290-2911.

42. Aly NY, Salmeen HN, Abo Lila RA, Nagaraja PA. Pantoea agglomerans blood stream infection in preterm neonates. Med Princ Pract. 2008;17:500-3.

43. Sengupta M, Banerjee S, Das NK, Guchhait P, Misra S. Early Onset Neonatal Septicaemia Caused by Pantoea agglomerans. J Clin Diagn Res. 2016;10(5):DD01-2.

44. 4Mahapatra A, Dhal S, Jena PP, Mohapatra A, Dash D, Padhee A. Neonatal septicaemia due to a rare bacterium: Pantoea agglomerans (case series). Paediatr Infect Dis. 2014;6:102-04.

45. Mehar V. Yadav D. Sanghvi J. Gupta N. Singh K. Pantoea dispersa: an unusual cause of neonatal sepsis. Braz J Infect Dis. 2013;17(6): 726-728.

46. Ministerio de Salud y Protección Social, Colciencias. Guía de práctica clínica. Recién nacido: sepsis neonatal temprana. Versión completa. Bogotá: Alianza CINETS; 2013. Guía No 6. 